$\mathrm{J}$ o u r n a l of

Mathematics

and Applications

No 35, pp 23-32 (2012)

\title{
The reticulation of residuated lattices induced by fuzzy prime spectrum
}

\author{
Shokoofeh Ghorbani
}

\author{
Submitted by: Jan Stankiewicz
}

\begin{abstract}
In this paper, we use the fuzzy prime spectrum to define the reticulation $(L(A), \lambda)$ of a residuated lattice $A$. We obtain some related results. In particular, we show that the lattices of fuzzy filters of a residuated lattice $A$ and $L(A)$ are isomorphic and the fuzzy prime spectrum of $A$ and $L(A)$ are homomorphic topological space.
\end{abstract}

AMS Subject Classification: 03G25, 54B35, 03E72.

Key Words and Phrases: Residuated lattice, Reticulation, Fuzzy filters, Fuzzy prime spectrum

\section{Introduction}

M. Ward and R.P. Dilworth [12] introduced the concept of residuated lattices as generalization of ideal lattices of rings. These algebras have been widely studied (See [1], [2] and [6]).

The reticulation was first defined by simmons([10]) for commutative ring and L. Leustean made this construction for BL-algebras ([7]). C. Mureson defined the reticulations for residuated lattices ([8]). The reticulation of an algebra $A$ is a pair $(L(A), \lambda)$ consisting of a bounded distributive lattice $L(A)$ and a surjective $\lambda: A \rightarrow L(A)([8])$. Hence we can transfer many properties between $A$ and $L(A)$.

The concept of fuzzy sets were introduced by Zadeh in 1965 ([13]). This concept was applied to residuated lattices and proposed the notions of fuzzy filters and prime fuzzy filters in a residuated lattice ([3], [4] and [14]). We defined and studied fuzzy prime spectrum of a residuated lattice in $([5])$.

In this paper, we use fuzzy prime spectrum to define the congruence relation $\cong$ on a residuated lattice $A$. Then we will show that $A / \cong$ is a bounded distributive lattice and $(A / \cong, \pi)$ is a reticulation of $A$. We will investigate some related results. Also, we obtain the relation between the reticulation of a residuated lattice induced by fuzzy prime spectrum and the reticulation of a residuated lattice which is defined in ([8]).

COPYRIGHT (c) by Publishing Department Rzeszów University of Technology P.O. Box 85, 35-959 Rzeszów, Poland 


\section{$2 \quad$ Preliminaries}

We recall some definitions and theorems which will be needed in this paper.

Definition 2.1. ([1], [11]) A residuated lattice is an algebraic structure $(A, \wedge, \vee, \rightarrow$ $, *, 0,1)$ such that

(1) $(A, \wedge, \vee, 0,1)$ is a bounded lattice with the least element 0 and the greatest element 1 ,

(2) $(A, *, 1)$ is a commutative monoid where 1 is a unit element,

(3) $x * y \leq z$ iff $x \leq y \rightarrow z$, for all $x, y, z \in A$.

In the rest of this paper, we denote the residuated lattice $(A, \wedge, \vee, *, \rightarrow, 0,1)$ by $A$.

Proposition 2.2. ([6], [11]) Let $A$ be a residuated lattice. Then we have the following properties: for all $x, y, z \in A$,

(1) $x \leq y$ if and only if $x \rightarrow y=1$,

(2) $x * y \leq x \wedge y \leq x, y$,

(3) $x *(y \vee z)=(x * y) \vee(x * z)$.

Definition 2.3. ([6], [11]) Let $F$ be a non-empty subset of a residuated lattice $A$. $F$ is called a filter if

(1) $1 \in F$,

(2) if $x, x \rightarrow y \in F$, then $y \in F$, for all $x, y \in A$.

$F$ is called proper, if $F \neq A$.

Theorem 2.4. ([6], [11]) A non-empty subset $F$ of a residuated lattice $A$ is a filter if and only if

(1) $x, y \in F$ implies $x * y \in F$,

(2) if $x \leq y$ and $x \in F$, then $y \in F$.

Definition 2.5. ([6]) Let $X$ be a subset of a residuated lattice $A$. The smallest filter of $A$ which contains $X$ is said to be the filter generated by $X$ and will be denoted by $<X>$.

Proposition 2.6. ([6]) Let $X$ be a non-empty subset of a residuated lattice $A$. Then $<X>=\left\{a \in A: a \geq x_{1} * \ldots * x_{n}\right.$ for some $\left.x_{1}, \ldots, x_{n} \in X\right\}$.

Definition 2.7. ([6], [11]) A proper filter $F$ of a residuated lattice $A$ is called prime filter, if for all $x, y \in A, x \vee y \in A$, implies $x \in A$ or $y \in A$.

Proposition 2.8. ([9]) (The prime filter theorem) Let $A$ be a residuated lattice, $F$ be a filter of $A$ and $a \in A \backslash F$. Then there exists a prime filter of $A$ that includes $F$ and does not contain $a$.

Definition 2.9. ([13]) Let $X$ be a non-empty subset. A fuzzy set in $X$ is a mapping 
$\mu: X \longrightarrow[0,1]$. For $t \in[0,1]$, the set $\mu_{t}=\{x \in X: \mu(x) \geq t\}$ is called a level subset of $\mu$. We call that $\mu$ is proper, if it has more two distinct values.

Definition 2.10. Let $X, Y$ be non-empty sets and $f: X \rightarrow Y$ be a function. Let $\mu$ be a fuzzy set in $X$ and $\nu$ be a fuzzy set in $Y$. Then $f(\mu)$ is a fuzzy set in $Y$ defined by

$$
f(\mu)(y)=\left\{\begin{array}{cll}
\sup \left\{\mu(x): x \in f^{-1}(y)\right\} & \text { if } & f^{-1}(y) \neq \emptyset \\
0 & \text { if } & f^{-1}(y)=\emptyset
\end{array}\right.
$$

for all $y \in Y$ and $f^{-1}(\nu)$ is a fuzzy set in $X$ defined by $f^{-1}(\nu)(x)=\nu(f(x))$ for all $x \in X$.

Definition 2.11. Let $X$ be a lattice. A fuzzy set $\mu$ is called a fuzzy lattice filter in $X$ if it satisfies: for all $x, y \in X$,

(1) $\mu(x) \leq \mu(1)$

(2) $\min \{\mu(x), \mu(y)\} \leq \mu(x \wedge y)$.

The set of all fuzzy lattice filter in $X$ is denoted by $\mathcal{F} \mathcal{L}(X)$.

Definition 2.12. ([3], [14]) Let $A$ be a residuated lattice. A fuzzy set $\mu$ is called a fuzzy filter in $A$ if it satisfies: for all $x, y \in A$,

$(f F 1) \mu(x) \leq \mu(1)$,

$(f F 4) \min \{\mu(x), \mu(x \rightarrow y)\} \leq \mu(y)$.

The set of all fuzzy filter in $A$ is denoted by $\mathcal{F}(A)$.

Theorem 2.13. ([3], [14]) Let $A$ be a residuated lattice. A fuzzy set $\mu$ in $A$ is a fuzzy filter if and only if it satisfies: for all $x, y \in A$,

( fF1) $x \leq y$ imply $\mu(x) \leq \mu(y)$,

$(f F 2) \min \{\mu(x), \mu(y)\} \leq \mu(x * y)$.

Proposition 2.14. ([3]) Let $\mu$ be a fuzzy filter of $A$. If $\mu(x \rightarrow y)=\mu(1)$, then $\mu(x) \leq \mu(y)$, for any $x, y \in A$.

Definition 2.15. Let $\mu$ be a fuzzy set in a residuated lattice $A$. The smallest fuzzy filter in $A$ which contains $\mu$ is said to be the fuzzy filter generated by $\mu$ and will be denoted by $<\mu>$.

Proposition 2.16. Let $\mu$ be a fuzzy set of a residuated lattice $A$. Then $\langle\mu\rangle(x)=$ $\sup \left\{\min \left\{\mu\left(a_{1}\right), \ldots, \mu\left(a_{n}\right)\right\}: x \geq a_{1} * \ldots * a_{n}\right.$ for some $\left.a_{1}, \ldots, a_{n} \in X\right\}$, for all $x \in A$.

Definition 2.17. ([5]) Let $\mu$ be a proper fuzzy filter in a residuated lattice $A . \mu$ is called a fuzzy prime filter if $\mu(x \vee y) \leq \max \{\mu(x), \mu(y)\}$ for all $x, y \in A$.

Theorem 2.18. ([5]) A proper subset $P$ of a residuated lattice $A$ is a prime filter of $A$ if and only if $\chi_{P}$ is a fuzzy prime filter in $A$. 
Theorem 2.19. ([5]) Let $A$ and $A^{\prime}$ be residuated lattices and $f: A \rightarrow A^{\prime}$ be an epimorphism. If $\mu$ is a fuzzy prime filter in $A$ which is constant on $\operatorname{ker}(f)$, then $f(\mu)$ is a fuzzy prime filter in $A^{\prime}$.

Theorem 2.20. ([5]) Let $A$ and $A^{\prime}$ be residuated lattices and $f: A \rightarrow A^{\prime}$ be a homomorphism. If $\nu$ is a fuzzy prime filter in $A^{\prime}$, then $f^{-1}(\nu)$ is a fuzzy prime filter in $A$.

Notation: ([5]) We shall denote the set of all fuzzy prime filter $\mu$ in a residuated lattice $A$ such that $\mu(1)=1$ by $F \operatorname{spec}(A)$. For each fuzzy set $\nu$ in $A$, define $\mathcal{C}(\nu)=\{\mu \in F \operatorname{spec}(A): \nu \leq \mu\}$. Let $\mu=\chi_{\{a\}}$ for $a \in A$. We shall denote $\mathcal{C}(\mu)$ by $\mathcal{C}(a)$ for all $a \in A$. Thus $\mathcal{C}(a)=\{\mu \in F \operatorname{spec}(A): \mu(a)=1\}$.

Proposition 2.21 ([5]) Let $\mu, \nu$ be fuzzy sets in a residuated lattice $A$ and $a, b \in A$. Then

(1) $\mu \leq \nu$ imply $\mathcal{C}(\nu) \subseteq \mathcal{C}(\mu) \subseteq F \operatorname{spec}(A)$.

(2) $\mathcal{C}\left(\bigcup_{i \in I} \nu_{i}\right)=\bigcap_{i \in I} \mathcal{C}\left(\nu_{i}\right)$.

(3) $\mathcal{C}(\mu) \cup \mathcal{C}(\nu) \subseteq \mathcal{C}(<\mu>\cap<\nu>)$.

(4) $\mathcal{C}(a \wedge b)=\mathcal{C}(a) \cup \mathcal{C}(b)$,

(5) $\mathcal{C}\left(\chi_{A}\right)=\bigcap_{a \in A} \mathcal{C}(a)$.

Theorem 2.22.([5]) Let $\mathcal{V}(a)=F \operatorname{spec}(A) \backslash \mathcal{C}(a)$ and $\mathcal{B}=\{\mathcal{V}(a): a \in A\}$. Then $\mathcal{B}$ is a base for a topology on $F \operatorname{spec}(A)$. The topological space $F \operatorname{spec}(A)$ is called fuzzy spectrum of $A$.

\section{The reticulation of residuated lattices}

Definition 3.1. Let $A$ be a residuated lattice. Define

$$
a \cong b \quad \text { if and only if } \quad \mathcal{C}(a)=\mathcal{C}(b),
$$

for all $a, b \in A$. Hence $a \cong b$ iff for any $\mu \in F \operatorname{spec}(A),(\mu(a)=1$ iff $\mu(b)=1)$.

Theorem 3.2. The relation $\cong$ is a congruence relation on a residuated lattice $A$ with respect to $*, \wedge$ and $\vee$.

Proof: It is clear that $\cong$ is an equivalence relation on $A$. Suppose that $a \cong b$ and $c \cong d$ where $a, b, c, d \in A$. We will show that $a * c \cong b * d, a \wedge c \cong b \wedge d$ and $a \vee c \cong b \vee d$. (1) Let $\mu \in \mathcal{C}(a * c)$. So $\mu(a * c)=1$. By Proposition 2.2 part (2) and Theorem 2.13, we have $1=\mu(a * c) \leq \mu(a), \mu(c)$. We get that $\mu(a)=\mu(c)=1$. By assumption, $\mu(b)=\mu(d)=1$. Since $b * d \leq b * d$, then $d \leq b \rightarrow(b * d)$ by Definition 2.1 part (3). We obtain that $1=\mu(d) \leq \mu(b \rightarrow b * d)$ by Theorem 2.13. Since $\mu$ is a fuzzy filter in $A$, we have $1=\min \{\mu(b), \mu(b \rightarrow b * d)\} \leq \mu(b * d)$. Then $\mu(b * d)=1$, that is $\mu \in \mathcal{C}(b * d)$. Hence $\mathcal{C}(a * c) \subseteq \mathcal{C}(b * d)$. Similarly, we can show that $\mathcal{C}(b * d) \subseteq \mathcal{C}(a * c)$. Therefore $a * c \cong b * d$. 
(2) Let $a \wedge c \cong b \wedge d$ and $\mu \in \mathcal{C}(a \wedge c)$. Thus $\mu(a \wedge c)=1$. Since $a \wedge c \leq a, c$, then $1=\mu(a \wedge c) \leq \mu(a), \mu(c)$ by Theorem 2.13. By assumption $\mu(b)=\mu(d)=1$. Since $\mu$ is a fuzzy filter in $A$ and $b * d \leq b \wedge d$, then $1=\min \{\mu(b), \mu(d)\} \leq \mu(b * d) \leq \mu(b \wedge d)$ by Theorem 2.13. Hence $\mu(b \wedge d)=1$ and then $\mathcal{C}(a \wedge c) \subseteq \mathcal{C}(b \wedge d)$. Similarly, we can show that $\mathcal{C}(b \wedge d) \subseteq \mathcal{C}(a \wedge c)$. Therefor $a \wedge c \cong b \wedge d$.

(3) Let $a \vee c \cong b \vee d$ and $\mu \in \mathcal{C}(a \vee b)$. Then $\mu(a \vee b)=1$. Since $\mu$ is a fuzzy prime filter in $A$, we have $\mu(a)=1$ or $\mu(b)=1$. By assumption $\mu(c)=1$ or $\mu(d)=1$. Hence $\mu(c \vee d)=\max \{\mu(c), \mu(d)\}=1$. We obtain that $\mu \in \mathcal{C}(c \vee d)$ and then $\mathcal{C}(a \vee b) \subseteq \mathcal{C}(c \vee d)$. Similarly, we can prove that $\mathcal{C}(c \vee d) \subseteq \mathcal{C}(a \vee b)$. Hence $a \vee c \cong b \vee d$.

Notation: Let $\cong$ be a the congruence relation on residuated lattice $A$ which is defined in Definition 3.1. For all $a \in A$, the equivalence class of $a$ is denoted by $[a]$, that is $[a]=\{b \in A: a \cong b\}$. The set of all equivalence classes is denoted by $A / \cong$.

Theorem 3.3. The algebra $(A / \cong, \wedge, \vee,[0],[1])$ is a bounded lattice, where

$$
[a] \vee[b]=[a \vee b] \text { and }[a] \wedge[b]=[a \wedge b]
$$

for all $a, b \in A$.

Proof: By Theorem 3.2, the operation $\wedge$ and $\vee$ are well defined. The rest of the proof is routine.

Example 3.4. Consider the residuated lattice $A$ with the universe $\{0, a, b, c, d, 1\}$. Lattice ordering is such that $0<a, b<c<1,0<b<d<1$ but $\{a, b\}$ and $\{c, d\}$ are incomparable. The operations of $*$ and $\rightarrow$ are given by the tables below :

\begin{tabular}{c|cccccc}
$*$ & 0 & $a$ & $b$ & $c$ & $d$ & 1 \\
\hline 0 & 0 & 0 & 0 & 0 & 0 & 0 \\
$a$ & 0 & $a$ & 0 & $a$ & 0 & $a$ \\
$b$ & 0 & 0 & 0 & 0 & $b$ & $b$ \\
$c$ & 0 & $a$ & 0 & $a$ & $b$ & $c$ \\
$d$ & 0 & 0 & $b$ & $b$ & $d$ & $d$ \\
1 & 0 & $a$ & $b$ & $c$ & $d$ & 1
\end{tabular}

\begin{tabular}{r|llllll}
$\rightarrow$ & 0 & $a$ & $b$ & $c$ & $d$ & 1 \\
\hline 0 & 1 & 1 & 1 & 1 & 1 & 1 \\
$a$ & $d$ & 1 & $d$ & 1 & $d$ & 1 \\
$b$ & $c$ & $c$ & 1 & 1 & 1 & 1 \\
$c$ & $b$ & $c$ & $d$ & 1 & $d$ & 1 \\
$d$ & $a$ & $a$ & $c$ & $c$ & 1 & 1 \\
1 & 0 & $a$ & $b$ & $c$ & $d$ & 1
\end{tabular}

Consider $0 \leq \nu_{1}(0)=\nu_{1}(a)=\nu_{1}(b)=\nu_{1}(c)<\nu_{1}(d)=\nu_{1}(1)=1$ and $0 \leq \nu_{2}(0)=$ $\nu_{2}(b)=\nu_{2}(d)<\nu_{2}(c)=\nu_{2}(c)=\nu_{2}(1)=1$. Then $F \operatorname{spec}(A)=\left\{\nu_{1}, \nu_{2}\right\}$. We obtain that $[0]=[b],[a]=[c]$. Therefore $A / \cong=\{[0],[a],[d],[1]\}$ where $[0]<[a],[d]<1$ but $\{[a],[d]\}$ are incomparable.

Lemma 3.5. Let $A$ be a residuated lattice and $a, b \in A$. Then

(i) $[a] \leq[b]$ if and only if $\mathcal{C}(b) \subseteq \mathcal{C}(a)$,

(ii) if $a \leq b$, then $[a] \leq[b]$,

(iii) $[a \wedge b]=[a * b]$. 
Proof: (i) By Theorem 3.3 and Proposition 2.21 parts (1) and (4), we have $[a] \leq[b]$ iff $[a] \wedge[b]=[a]$ iff $[a \wedge b]=[a]$ iff $\mathcal{C}(a)=\mathcal{C}(a \wedge b)=\mathcal{C}(a) \cup \mathcal{C}(b)$ iff $\mathcal{C}(b) \subseteq \mathcal{C}(a)$.

(ii) If $a \leq b$, then $\mathcal{C}(a) \subseteq \mathcal{C}(b)$. We obtain that $[a] \leq[b]$ by (i).

(iii) We will show that $\mathcal{C}(a * b)=\mathcal{C}(a \wedge b)$. Let $\mu \in \mathcal{C}(a * b)$. Then $\mu(a * b)=1$. By Proposition 2.2 part (2) and Theorem 2.13, $\mu(a * b) \leq \mu(a \wedge b)$. We get that $\mu(a \wedge b)=1$ and then $\mu \in \mathcal{C}(a \wedge b)$. Hence $\mathcal{C}(a * b) \subseteq \mathcal{C}(a \wedge b)$.

Conversely, let $\mu \in \mathcal{C}(a \wedge b)$. Then $\mu(a \wedge b)=1$. Since $a \wedge b \leq a, b$, then $\mu(a)=\mu(b)=1$ by Theorem 2.13. Since $b \leq a \rightarrow(a * b)$ and $\mu$ is a fuzzy filter in $A$,

$$
1=\min \{\mu(a), \mu(b)\} \leq \min \{\mu(a), \mu(a \rightarrow(a * b))\} \leq \mu(a * b) .
$$

Hence $\mu(a * b)=1$ and then $\mu \in \mathcal{C}(a * b)$. We get that $\mathcal{C}(a \wedge b) \subseteq \mathcal{C}(a * b)$. Therefor $[a \wedge b]=[a * b]$.

Theorem 3.6. The bounded lattice $(A / \cong, \wedge, \vee,[0],[1])$ is distributive.

Proof: Let $a, b, c \in A$. By Lemma 3.5 and Proposition 2.2 part (3),

$$
\begin{aligned}
{[a] \wedge([b] \vee[c])=} & {[a \wedge(b \vee c)]=[a *(b \vee c)] } \\
& =[(a * b) \vee(a * c)]=[a * b] \vee[a * c] \\
& =[a \wedge b] \vee[a \wedge c]=([a] \wedge[b]) \vee([a] \wedge[c])
\end{aligned}
$$

Definition 3.7. Let $A$ be a residuated lattice and $\pi: A \rightarrow A / \cong$ be that canonical surjective map defined by $\pi(a)=[a]$. Then $(A / \cong, \pi)$ is called the reticulation of residuated lattice induced by fuzzy filters.

Lemma 3.8. Let $A_{1}$ and $A_{2}$ be residuated lattices and $f: A_{1} \rightarrow A_{2}$ be a homomorphism of residuated lattices. Then $\mathcal{C}(a)=\mathcal{C}(b)$ implies $\mathcal{C}(f(a))=\mathcal{C}(f(b))$, for any $a, b \in A_{1}$.

Proof: Suppose that $\mathcal{C}(a)=\mathcal{C}(b)$ where $a, b \in A_{1}$ and $\nu \in \mathcal{C}(f(a))$. Then $\nu \in$ $F \operatorname{spec}\left(A_{2}\right)$ and $\nu(f(a))=1$. By Theorem 2.20, we have $f^{-1}(\nu) \in F \operatorname{spec}\left(A_{1}\right)$ and $f^{-1}(\nu)(a)=\nu(f(a))=1$. Thus $f^{-1}(\nu) \in \mathcal{C}(a)=\mathcal{C}(b)$. We get that $\nu(f(b))=$ $f^{-1}(\nu)(b)=1$ and then $\nu \in \mathcal{C}(f(b))$. Hence $\mathcal{C}(f(a)) \subseteq \mathcal{C}(f(b))$. Similarly, we can show that $\mathcal{C}(f(b)) \subseteq \mathcal{C}(f(a))$

In the following theorem, we will define a functor from the category of residuated lattices to the category of bounded distributive lattices.

Theorem 3.9. Let $A_{1}$ and $A_{2}$ be residuated lattices and $f: A_{1} \rightarrow A_{2}$ be a homomorphism of residuated lattices. Then $\bar{f}: A_{1} / \cong \rightarrow A_{2} / \cong$ is defined by $\bar{f}([a])=[f(a)]$ is a homomorphism of lattices.

Proof: Let $[a]=[b]$. By Lemma 3.5 part (i), we obtain that $\mathcal{C}(a)=\mathcal{C}(b)$. By Lemma $3.8, \mathcal{C}(f(a))=\mathcal{C}(f(b))$. We have $[f(a)]=[f(b)]$ by Lemma 3.5 part (i). So $\bar{f}$ is well defined. Now, Let $a, b \in A_{1}$. Since $f$ is a homomorphism of residuated lattices, then 


$$
\bar{f}([a] \wedge[b])=\bar{f}([a \wedge b])=[f(a \wedge b)]=[f(a)] \wedge[f(b)]=\bar{f}([a]) \wedge \bar{f}([b]) .
$$

Similarly, we can show that $\bar{f}([a] \vee[b])=\bar{f}([a]) \vee \bar{f}([b])$. Also, $\bar{f}([0])=[f(0)]=[0]$ and $\bar{f}([1])=[f(1)]=[1]$. Hence $\bar{f}$ is a homomorphism of lattices.

Lemma 3.10. Let $\mu$ be a fuzzy filter in a residuated lattice $A$ and $a, b \in A$ such that $[a]=[b]$. Then $\mu(a)=\mu(b)$.

Proof: Suppose that $\mu$ is a fuzzy filter in $A$ such that $\mu(a) \neq \mu(b)$. Then $\mu(a)<\mu(b)$ or $\mu(b)<\mu(a)$. Let $\mu(a)<\mu(b)$. Put $F=\{x \in A: \mu(x) \geq \mu(b)\}$, i.e. $F=\mu_{\mu(b)}$. Hence $F$ is a filter of $A$ such that $a \notin F$. Define $J=<F \cup\{b\}>$. Then $J$ is a filter of $A$. We shall show that $a \notin J$. Suppose that $a \in J$. By Proposition 2.6, there exist $y_{1}, \ldots, y_{n} \in F \cup\{b\}$ such that $y_{1} * \ldots * y_{n} \leq a$. If $y_{i}=b$ for some $1 \leq i \leq n$, then $y_{1} * \ldots * y_{i-1} * y_{i+1} \ldots * y_{n} * b \leq a$. Hence $y_{1} * \ldots * y_{i-1} * y_{i+1} \ldots * y_{n} \leq b \rightarrow a$. Since $F$ is a filter, we have $b \rightarrow a \in F$, that is $\mu(b \rightarrow a) \geq \mu(b)$. So $\mu(b)=\min \{\mu(b), \mu(b \rightarrow a)\} \leq \mu(a)$ which is a contradiction. Now, suppose that $y_{i} \in F$ for all $1 \leq i \leq n$. Thus $y_{1} * \ldots * y_{n} \in F$. We get that $a \in F$ which is a contradiction. Hence $a \notin J$ and $J$ is a proper filter. By Proposition 2.8, there exists a prime filter $P$ such that $J \subseteq P$ and $a \notin P$. By Theorem 2.18, $\chi_{P}$ is a fuzzy prime filter in $A$ such that $\chi_{P}(b)=1$ and $\chi_{P}(a) \neq 1$. We obtain that $\chi_{P} \in \mathcal{C}(b)$ but $\chi_{P} \notin \mathcal{C}(a)$ which is a contradiction. Hence $\mu(a)=\mu(b)$.

Theorem 3.11. Let $\mu$ be a fuzzy filter in a residuated lattice $L$. Then $\pi(\mu)$ is a

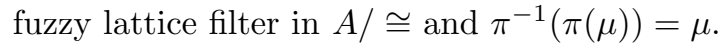

Proof: Let $[a],[b] \in A / \cong$. Then $\pi(a)=[a]$ and $\pi(b)=[b]$. Since $\pi$ is a homomorphism, we have $[a] \wedge[b]=[a \wedge b]=\pi(a \wedge b)$. We get that $a \wedge b=\pi^{-1}(x \wedge y)$. We have

$$
\begin{aligned}
\pi(\mu)([a] \wedge[b])= & \sup \left\{\mu(z): z \in \pi^{-1}[a \wedge b]\right\} \\
& \geq \sup \left\{\mu(x \wedge y): x \in \pi^{-1}([a]), y \in \pi^{-1}([b])\right\} \\
& =\sup \left\{\min \{\mu(x), \mu(y)\}: x \in \pi^{-1}([a]), y \in \pi^{-1}([b])\right\} \\
& =\min \left\{\sup \left\{\mu(x): x \in \pi^{-1}([a])\right\}, \sup \left\{\mu(b): y \in \pi^{-1}([b])\right\}\right\} \\
& =\min \{\pi(\mu)(a), \pi(\mu)(b)\} .
\end{aligned}
$$

Let $[a] \leq[b]$. Then $\pi(a) \leq \pi(b)$. We shall show that $\pi(\mu)([a]) \leq \pi(\mu)([b])$. Suppose that $\pi(\mu)[a]>\pi(\mu)[b]$. Then there exists $x_{0} \in \pi^{-1}([a])$ such that $\pi\left(x_{0}\right)=a$ and $\mu\left(x_{0}\right)>\sup \left\{\mu(y): y \in \pi^{-1}(b)\right\}$. We have $\mu(y) \leq \mu\left(x_{0}\right)$ for all $y \in \pi^{-1}(b)$. Let $y \in \pi^{-1}(b)$ be arbitrary. Since $\pi$ is a lattice homomorphism, then $[b]=[a] \vee[b]=$ $\pi\left(x_{0}\right) \vee \pi(y)=\pi\left(x_{0} \vee y\right)$. Hence $x_{0} \vee y \in \pi^{-1}(b)$. Therefore $\mu\left(x_{0} \vee y\right)<\mu\left(x_{0}\right)$. By Definition 2.17, $\mu\left(x_{0} \vee y\right) \geq \max \left\{\mu\left(x_{0}\right), \mu(y)\right\}=\mu\left(x_{0}\right)$ which is a contradiction. Hence $\pi(\mu)$ is a fuzzy lattice filter in $A / \cong$. By Lemma 3. 10, we have $\pi^{-1}(\pi(\mu))(a)=\pi(\mu)(\pi(a))=\pi(\mu)[a]=\sup \left\{\mu(x): x \in \pi^{-1}([a])\right\}=\sup \{\mu(x):$ $\pi(x)=[a]\}=\sup \{\mu(x):[x]=[a]\}=\mu(a)$. 
Theorem 3.12. Let $\nu$ be a fuzzy lattice filter in a lattice $A / \cong$. Then $\pi^{-1}(\nu)$ is a fuzzy filter in $A$ and $\pi\left(\pi^{-1}(\nu)\right)=\nu$.

Proof: Let $x, y \in A$. By Lemma 3.5 part (iii), we have $\pi^{-1}(\nu)(x * y)=\nu(\pi(x * y))=$ $\nu([x * y])=\nu([x \wedge y])=\nu([x] \wedge[y]) \geq \min \{\nu([x]), \nu([y])\}=\min \left\{\pi^{-1}(\nu)(x), \pi^{-1}(\nu)(y)\right\}$. Suppose that $x \leq y$. By Lemma 3.5 part (ii), we have $[x] \leq[y]$. Since $\nu$ is a fuzzy lattice filter in $A / \cong$, we have $\nu([x]) \leq \nu([y])$, that is $\pi^{-1}(\nu)(x) \leq \pi^{-1}(\nu)(y)$. By Lemma 3.10, we obtain that $\pi\left(\pi^{-1}(\nu)\right)[x]=\sup \left\{\pi^{-1}(\nu)(y): y \in \pi^{-1}([x])\right\}=\sup \left\{\pi^{-1}(\nu)(y)\right.$ : $\pi(y)=[x]\}=\sup \left\{\pi^{-1}(\nu)(y):[y]=[x]\right\}=\nu([x])$

Proposition 3.13. Let $\mu$ and $\nu$ be fuzzy filters in a residuated lattice $A$. Then $\nu \leq \mu$ if and only if $\pi(\nu) \leq \pi(\mu)$.

Proof: Suppose that $\nu \leq \mu$. Then $\pi(\nu)([x])=\sup \left\{\nu(y): y \in \pi^{-1}([x])\right\} \leq \sup \{\mu(y)$ : $\left.y \in \pi^{-1}([x])\right\}=\pi(\mu)([x])$. Conversely, let $\pi(\nu) \leq \pi(\mu)$. Then $\nu(a)=\pi^{-1}(\pi(\nu))(a)=$ $\pi(\nu)(\pi(a)) \leq \pi(\mu)(\pi(a))=\pi^{-1}(\pi(\mu))(a)=\mu(a)$.

Theorem 3.14. There is a lattice isomorphism between the lattices $\mathcal{F}(A)$ and $\mathcal{F} \mathcal{L}(A / \cong)$.

Proof: Define $\varphi: \mathcal{F}(A) \rightarrow \mathcal{F} \mathcal{L}(A / \cong)$ by $\varphi(\mu)=\pi(\mu)$ and $\psi: \mathcal{F}(L / \equiv) \rightarrow \mathcal{F}(L)$ by $\psi(\nu)=\pi^{-1}(\nu)$. By Theorems 3.11 and $3.12 \varphi$ and $\psi$ are well defined and bijection. By the above Proposition $\varphi$ is a lattice homomorphism. Hence $\phi$ is an isomorphism of lattices.

Theorem 3.15. Let $\mu$ be a fuzzy prime filter in a residuated lattice $A$. Then $\pi(\mu)$ is a fuzzy prime filter in $A / \cong$.

Proof: Since $\mu$ is a fuzzy prime filter in $A$, then $\mu$ is proper. So $\mu(0) \neq \mu(1)$. By Lemma 3.10, $\pi(\mu)(0)=\sup \left\{\mu(x): x \in \pi^{-1}([0])\right\}=\sup \{\mu(x):[x]=[0]\}=\mu(0)=0$ and $\pi(\mu)(1)=\sup \left\{\mu(x): x \in \pi^{-1}([1])\right\}=\sup \{\mu(x):[x]=[1]\}=\mu(1)=1$. Hence $\pi(\mu)$ is proper. We have $\pi(\mu)([x \vee y])=\sup \left\{\mu(z): z \in \pi^{-1}([x \vee y])\right\}=\sup \{\mu(x)$ : $[z]=[x \vee y]\}=\mu(x \vee y)=\max \{\mu(x), \mu(y)\}$ Also, we have $\pi(\mu)[x]=\sup \{\mu(a): a \in$ $\left.\pi^{-1}([x])\right\} .=\sup \{\mu(a):[a]=[x]\}=\mu(x)$. Similarly, we can show that $\pi(\mu)[y]=\mu(y)$. We obtain that $\pi(\mu)(x \vee y)=\mu(x \vee y)=\max \{\mu(x), \mu(y)\}=\max \{\pi(\mu)(x), \pi(\mu)(y)\}$ and then $\pi(\mu)$ is a fuzzy prime filter in $A / \cong$

Theorem 3.16. Let $\nu$ be a fuzzy prime filter in a lattice $A / \cong$. Then $\pi^{-1}(\nu)$ is a fuzzy prime filter in $A$.

Proof: By assumption $\nu$ is proper. Hence $\nu([0]) \neq \nu([1])$. We have $\pi^{-1}(\nu)(0)=$ $\nu(\pi(0))=\nu([0])$ and $\pi^{-1}(\nu)(1)=\nu(\pi(1))=\nu([1])$. Hence $\pi^{-1}(\nu)(0) \neq \pi^{-1}(\nu)(1)$. That is $\pi^{-1}(\nu)$ is proper. Also, we have

$\pi^{-1}(\nu)(x \vee y)=\nu(\pi(x \vee y))=\nu([x \vee y])=\nu([x] \vee[y])=\max \{\nu([x]), \nu([y])\}=$ $\max \left\{\pi^{-1}(\nu)(x), \pi^{-1}(\nu)(y)\right\}$. 
Theorem 3.17. There exists a homomorphism between topological Space Fspec $(A)$ and $\operatorname{Fspec}(A / \cong)$.

Proof: Consider $\varphi$ in Theorem 3.14. The restriction $\varphi$ to $F \operatorname{spec}(A)$ is denoted by $\bar{\varphi}$. By Theorems 3.15 and 3.16, $\bar{\varphi}: F \operatorname{spec}(A) \rightarrow F \operatorname{spec}(A / \equiv)$ is a bijective. We will show that $\bar{\varphi}$ is continuous and closed. Let $\mathcal{C}([a])$ be an arbitrary closed base set. Then

$$
\begin{aligned}
\bar{\varphi}^{-1}(\mathcal{C}([a]) & =\{\mu \in F \operatorname{spec}(A): \bar{\varphi}(\mu) \in \mathcal{C}([a])\} \\
& =\{\mu \in F \operatorname{spec}(A): \pi(\mu) \in \mathcal{C}([a])\} \\
& =\{\mu \in F \operatorname{spec}(A): \pi(\mu)[a]=1\} \\
& =\{\mu \in F \operatorname{spec}(A): \mu(a)=1\}=\mathcal{C}(a)
\end{aligned}
$$

Hence $\varphi$ is continuous. Also, we have

$$
\begin{aligned}
\bar{\varphi}(\mathcal{C}(a))= & \{\varphi(\mu): \mu \in F \operatorname{spec}(A), \mu \in \mathcal{C}(a)\} \\
= & \{\pi(\mu): \mu \in F \operatorname{spec}(A), \mu \in \mathcal{C}(a)\} \\
& \{\pi(\mu): \mu \in F \operatorname{spec}(A), \mu(a)=1\} \\
& =\{\nu \in F \operatorname{spec}(A / \cong): \nu([a])=1\}=\mathcal{C}([a]) .
\end{aligned}
$$

Hence $\varphi$ is closed.

Let $A$ be a residuated lattice. For any $a, b \in A$ define $a \equiv b$ iff for any $P \in \operatorname{Spec}(A)$, $(a \in P$ iff $b \in P)$. Then $\equiv$ is a congruence relation on $A$ respect to $*, \wedge$ and $\vee$. Let us denot by $\bar{a}$ the equivalence class of $a \in A$ and let $A / \equiv$ be the quotient set. We denote $\lambda: A \rightarrow A / \equiv$ the canonical surjective defined by $\lambda(a)=\bar{a}$. Then $(A / \equiv, \vee, \wedge, 0,1)$ is a bounded distributive lattice and $(A / \equiv, \lambda)$ is a reticulation of $A$ (See [8]).

Theorem 3.18. Let $A$ be a residuated lattice. Then the congruence relation $\cong$ is equal to the congruence relation $\equiv$ on $A$.

Proof: Let $a, b \in A$ such that $a \cong b$. We have $(\mu(a)=1$ iff $\mu(b)=1)$ for any $\mu \in F \operatorname{spec}(A)$. Suppose that $P \in \operatorname{Spec}(A)$. By Theorem 2.18, $\chi_{P}$ is a fuzzy prime filter. Hence $\chi_{P}(a)=1$ iff $\chi_{P}(b)=1$. We get that $a \in P$ iff $b \in P$. Hence $a \equiv b$ and then $\cong \subseteq \equiv$.

Conversely, let $a \equiv b$ and $\mu \in F \operatorname{spec}(A)$ such that $\mu(a)=1$. We get that $a \in \mu_{1}$ and $\mu_{1}$ is a proper filter of $A$. Hence $\mu_{1} \in \operatorname{Spec}(A)$. Since $a \equiv b$, then we have $b \in \mu_{1}$. We obtain that $\mu(b)=1$. Similarly, we can prove that if $\mu(b)=1$, then $\mu(b)=1$. So $a \cong b$. Therefor $\equiv \subseteq \cong$

\section{References}

[1] T.S. Blyth, M. F. Janowitz, Residuation Theory, Pergamon Press, 1972. 
[2] K. Blount, C. Tsinakies, The structure of residuated lattices, Internat. J. Algebra Comput., 13(2003), No. 4, 437-461.

[3] Sh. Ghorbani, A. Hasankhani, Fuzzy Convex Subalgebras of Commutative Residuated Lattices, Iran. J. Fuzzy Syst., Vol. 7, No. 2(2010), 41-54.

[4] Sh. Ghorbani, A. Hasankhani, Uniform topology on a residuated lattice induced by fuzzy filters, Int. Rev. Fuzzy Math. 4(2009), 45-62.

[5] Sh. Ghorbani, Fuzzy spectrum of residuated lattices, Journal of Advanced Research in Pure Mathematics 3(2011), 56 - 68.

[6] T. Kowalski, H. Ono, Residuated lattices: An algebraic glimpse at logics without contraction, monograph, March, 2001.

[7] L. Leustean, The prime and maximal spectra and the reticulation of BL- algebras, Cent. Eur. J. Math. , No. 3 (2003), 382-397.

[8] C. Muresan, The reticulation of a residuated lattice, Bull. Math. Soc. Sci. Math. Roumania, Tome 51(99) No. 1, (2008), 47-65.

[9] D. Piciu, Algebras of fuzzy logic, Editura Universitaria, Craiova, 2007.

[10] H. Simmons, Reticulated rings, J. Algebra 66(1980), 169-192.

[11] E. Turunen, Mathematics behind fuzzy logic, Advances in Soft Comput- ing, Physica-Verlag, Heidelberg, 1999.

[12] M. Ward, R. P. Dilworth, Residuated lattices, Trans. Amer. Math. Soc. 45(1939), 335-354.

[13] L. A. Zadeh, Fuzzy sets, Inf. and Control 8(1965), 338-353.

[14] J. L. Zhang, H. J. Zhou, Fuzzy filters on the residuated lattices, New Math. Nat. Comput. 1(2006), 11-28.

\section{DOI: $10.7862 / \mathrm{rf} .2012 .2$}

\section{Shokoofeh Ghorbani}

email: sh.ghorbani@uk.ac.ir

Department of Mathematics,

Bam Higher Education Complexes,

Kerman, Iran

Received 14.04.2011, Revisted 26.01.2012 